\title{
EL PAPEL ASIGNADO A LA EDUCACIÓN SANITARIA EN LA ESPAÑA DE LA TRANSICIÓN DEMOCRÁTICA*
}

\author{
The role assigned to health education in the Spain \\ of the Democratic Transition
}

\section{Enrique Perdiguero-Gil ${ }^{\alpha}$ y Eduardo Bueno Vergara ${ }^{\beta}$}

Fecha de recepción: 31/03/2021 • Fecha de aceptación: 08/08/2021

Resumen. En España, a mediados de la década de los años setenta del pasado siglo, se discutieron diversos proyectos de reforma sanitaria que pretendían cambiar el desfasado sistema sanitario heredado del franquismo. En todas estas propuestas, pero en especial en las provenientes de los partidos de izquierda, se asignó un papel primordial a la educación sanitaria de la población. En un sistema sanitario que pretendía defender la salud en vez de curar la enfermedad, las conductas de la población en relación con los factores sociales y económicos que generaban enfermedades resultaban cruciales. Asimismo, la educación sanitaria se concibió como un medio para que la población participase en la planificación, gestión y evaluación de los servicios sanitarios. Estas afirmaciones genéricas supusieron, en muchas ocasiones, versiones ingenuas y clásicas de la educación sanitaria, similares a las propuestas por el gobierno. Frecuentemente se defendió el papel de la educación sanitaria en la racionalización del uso de los servicios sanitarios. Los proyectos de reforma sanitaria tendieron a establecer la hegemonía de lo sanitario frente a lo educativo. Algunas propuestas más diferenciadas dieron paso a acciones

\footnotetext{
* Este trabajo ha sido financiado por los proyectos «De la propaganda sanitaria a la educación para la salud: ideología, discursos y saberes en la España de Franco (1939-1975)» HAR2012-34588 (MINECO), «Reforma sanitaria y promoción de la salud en el tardofranquismo y la transición democrática: nuevas culturas de la salud, la enfermedad y la atención» HAR2015-64150-C2-1-P (MINECO/FEDER) y por la red NISALDes, RED2018-102413-T (Ministerio de Ciencia, Innovación y Universidades).

${ }^{\alpha}$ Instituto Interuniversitario López Piñero, Sede Universidad Miguel Hernández de Elche, N-332, km. 87, s/n, 03550 Sant Joan d'Alacant, Alicante, España. quique@umh.es (D) http://orcid.org/00000003-0870-3512

^ Instituto Interuniversitario López Piñero, Sede Universidad Miguel Hernández de Elche, N-332, km. 87, s/n, 03550 Sant Joan d'Alacant, Alicante, España. ebueno@umh.es (D) http://orcid.org/00000001-7185-6162
}

Cómo citar este artículo: Perdiguero-Gil, Enrique y Bueno Vergara, Eduardo. «El papel asignado a la educación sanitaria en la España de la Transición Democrática». Historia y Memoria de la Educación 15 (2022): 137-169 
más concretas a nivel local que fueron desarrollando la educación para la salud que se llevaría a cabo en las décadas siguientes.

Palabras clave: Educación sanitaria; Franquismo; Transición democrática; Reforma sanitaria; España.

Abstract. In the mid-1970s, several health reform projects were discussed in Spain for the purpose of changing the outdated health system inherited from Francoism. In all these proposals, but especially in those promoted by the leftist parties, a primary role was assigned to the health education of the general population. In a health system that sought to defend health rather than cure diseases, the behaviour of the population in relation to the social and economic factors that generated diseases was crucial. Furthermore, health education was conceived as a way for the population to participate in the planning, management, and evaluation of health services. These generic statements were, on many occasions, naive and classic versions of health education, similar to those proposed by the government. The role of health education in rationalizing the use of health services was frequently invoked. Health reform projects tended to establish the hegemony of health workers over teachers. Some more specific proposals gave way to more concrete actions at a local level that led to the development of the model of health education that was carried out in the following decades.

Keywords: Health education; Francoism; Health reform; Spain.

\section{INTRODUCCIÓN}

En 1970, en uno de los primeros textos que indicaban la necesidad de reformar el sector sanitario construido durante el franquismo, se afirmaba lo siguiente: "La deficiente educación sanitaria de la población española es una de las mayores taras de nuestra sanidad». ${ }^{1}$ Pocos años después, en 1976, Josep Artigas y Helios Pardell, al criticar la política sanitaria catalana, señalaban que la «[...] Promoció de la salut. Basada, fonamentalment, en l'educació sanitària [...], com la resta de la tasca educadora, ha estat deixada de cantó sistemàticament». ${ }^{2}$ La afirmación

\footnotetext{
${ }_{1}^{1}$ El entrecomillado aparece en el Preámbulo al número extraordinario de Cuadernos para el Dialogo, publicado en mayo de 1970, dedicado a «La crisis de la medicina en España. Médicos, Medicina, Sociedad», s.p.

2 Josep Artigas y Hèlios Pardell, «Estructura actual de la sanitat. Defectes de l'estructura i política sanitaria», en Xè Congrés de Metges i Biòlegs de Llengua Catalana. II Ponència. Funció social de la medicina, (Barcelona: Acadèmia de Ciències Médiques de Catalunya i de Balears. Societat Catalana de Biologia, 1976), 210. Este tipo de congresos venía celebrándose desde 1913 y se interrumpieron con la Guerra Civil. El noveno había tenido lugar también en Perpiñán, en 1936. El texto de Artigas
} 
formaba parte de una ponencia presentada al Xè Congrés de Metges i Biòlegs en Llengua Catalana, celebrado en Perpiñán. Aunque referida a Cataluña, podía hacerse extensiva al conjunto de España, pues los elementos que la justificaban implicaban a todo el país. ${ }^{3}$

Una década después, el panorama era muy diferente y la educación sanitaria había pasado a ocupar un lugar privilegiado dentro de los proyectos de reforma sanitaria, a juzgar por la nota editorial de uno de los Quaderns del Centre d'Anàlisi i Programes Sanitaris (CAPS):4

La Educación para la Salud se ha convertido, con el tiempo, en uno de los leitmotiv [sic] de toda reforma sanitaria. No hay discurso programático sobre política de salud que no incluya una expresa referencia a la Educación Sanitaria, si bien, en ocasiones, es tan sólo el tópico que, como otros varios, los políticos quieren no olvidar.

Pero hoy sabemos que la Educación para la salud es un tema bastante más complejo de lo que se creía hace unos años. ${ }^{5}$

El objetivo de este trabajo es analizar el papel que, en el contexto de los debates sobre la reforma sanitaria, se asignó a la educación sanitaria, pues no solo se le otorgó un valor estrictamente sanitario, sino catalizador de un nuevo modelo de sociedad, tal y como afirmaba Yuste en 1971:

\footnotetext{
y Pardell forma parte de la ponencia sobre la función social de la medicina que tendría una gran influencia en los debates posteriores sobre la reforma sanitaria. Artigas, abogado, tuvo un papel destacado en la sanidad catalana; Pardell, médico, se dedicó a la actividad clínica.

${ }^{3}$ Hemos escogido «educación sanitaria» como el término más utilizado en los proyectos que se analizan en este artículo. En los años veinte y treinta se usó "propaganda sanitaria» y a finales de los sesenta y en los setenta comenzaron a emplearse «educación para la salud» y «promoción de la salud». Hay notorias diferencias conceptuales entre ellos, pero su uso indistinto fue frecuente. A nivel internacional véase Julia Vanel, «De l'éducation sanitaire à la promotion de la santé: Enjeux et organisation des savoirs au coeur de l'action publique sanitaire (internationale)». (Thèse de doctorat, Université Paris Saclay (COmUE), 2006).

${ }^{4}$ El CAPS, fundado en Barcelona en 1983, tiene como objeto contribuir, a través del análisis pluridisciplinar de la sanidad, al intercambio de ideas, a la reflexión y a la investigación de todo lo relacionado con la salud. Es el heredero del Gabinet d'Assessoria i Promoció de la Salut, puesto en marcha en 1976 en el seno del Colegio de Médicos de Barcelona.
}

5 Centre d'Anàlisi i Programes Sanitaris, «Nota editorial», en ed. Centre d'Anàlis i Programes Sanitaris, Tendencias actuales en educación sanitaria, (Barcelona: Centre d'Anàlis i Programes Sanitaris, 1987), 5. 
[...] la educación sanitaria, a la vez que una actividad sanitaria, es una filosofía del desarrollo individual y comunitario, que conecta con las preocupaciones democráticas y de promoción de los pueblos y sus comunidades, y que considera que la labor en pro de la salud es el primer bien de cualquier hombre por encima de razones o justificaciones de tipo económico. ${ }^{6}$

En la misma línea, el texto del CAPS, indicaba que la educación sanitaria tenía que ver con:

[...] comprender lo trascendente que el rol protagonista del ciudadano puede tener para mejorar o empeorar la calidad de vida y las espectativas [sic] de salud [...] si se consiguiera que el ciudadano fuera protagonista de su vida y de la historia de la comunidad, probablemente se hallaría la fórmula mágica que permitiría una eficaz política de prevención. ${ }^{7}$

El interés del tema objeto de estudio radica en analizar cómo - y con qué características - las actividades educativas relacionadas con la salud, fuera del ámbito escolar, tuvieron un papel fundamental en los discursos sobre la reforma del sistema sanitario heredado del franquismo, una necesidad sobre la que había un consenso general desde inicios de los años setenta. Las fuentes que hemos utilizado son, fundamentalmente, documentos oficiales, monografías, actas de congresos y artículos de revista publicados desde el inicio de la Dictadura pero, sobre todo, en los años setenta e inicios de los ochenta del pasado siglo. Aunque, con frecuencia, el debate sobre la reforma sanitaria se circunscribe a los años que median entre la victoria en las elecciones generales de 1982 del Partido Socialista Obrero Español (PSOE) y la aprobación de la Ley General de Sanidad (LGS) en 1986, lo cierto es que se pueden encontrar intentos de reforma desde los años sesenta -la mayor parte de ellos fracasados- y, desde luego, abundantes debates al respecto en los años inmediatamente anteriores y posteriores a la muerte del dictador. ${ }^{8}$ Nos

\footnotetext{
${ }^{6}$ Francisco Javier Yuste Grijalba, La educación sanitaria (Madrid: Marsiega, 1971), 13. Yuste, psiquiatra y funcionario de Sanidad Nacional, fue senador y diputado del PSOE y colaboró con Ernest Lluch en la elaboración de la Ley General de Sanidad (LGS) de 1986.

7 Centre d'Anàlisi i Programes Sanitaris, «Nota editorial», 5.

${ }^{8}$ Enrique Perdiguero-Gil y Josep M Comelles, «The Roots of the health Reform in Spain», en Health Care and Government Policy, ed. Laurinda Abreu, (Évora: Publicações do Cidehus, 2019), 10.4000/
} 
vamos a centrar precisamente en estos últimos, tras resumir lo ocurrido en el franquismo, pues los avatares de la gestación de la LGS en la primera legislatura socialista son más conocidos. ${ }^{9}$ En esos años se dieron dos circunstancias relevantes. La primera, el reconocimiento del derecho a la protección de la salud por la Constitución Española de 1978, en cuyo artículo 43 se especificaba: «Los poderes públicos fomentarán la educación sanitaria, la educación física y el deporte. Asimismo, facilitarán la adecuada utilización del ocio». La segunda es la celebración en Alma-Ata (entonces Unión Soviética) de la Conferencia Internacional sobre Atención Primaria de Salud que otorgó un papel crucial a la educación sanitaria. ${ }^{10}$

Nuestro objeto de estudio es la educación, pero nuestro enfoque va a centrarse en el ámbito sanitario. Los médicos reclamaron para sí la hegemonía en la educación sanitaria de la población, aun reconociendo la imprescindible colaboración del mundo educativo. En lo referido a lo que se realizó en este último, remitimos al artículo de Terrón y Hurtado de este mismo monográfico.

Para desarrollar nuestros argumentos dedicaremos un primer apartado, con el fin de conocer el punto de partida de las propuestas reformadoras, a la educación sanitaria de la población realizada desde instancias oficiales durante el franquismo. En un segundo apartado analizaremos el papel asignado a la educación sanitaria en los proyectos que pretendieron crear un servicio nacional de salud que dejase atrás la sanidad franquista. En el tercer apartado indicaremos qué se entendió por educación sanitaria en el contexto reformista. Concluiremos con un balance sobre lo que significó la educación sanitaria en la reforma sanitaria y años posteriores.

\footnotetext{
books.cidehus.8327. Esteban Rodríguez Ocaña y Rosa Ballester Añón, «El Informe del consultor de la OMS Fraser Brockington de 1967 en el contexto del reformismo sanitario franquista», Dynamis 39, n. 2 (2019): 477-496, https://doi.org/10.30827/dynamis.v39i2.9845. Enrique Perdiguero-Gil y Josep M. Comelles, «The defence of health. The debates on health reform in 1970s Spain», Dynamis 39, n. 1 (2019): 45-72, https://doi.org/10.30827/dynamis.v39i1.8666. José Martínez-Pérez y Enrique Perdiguero-Gil, eds., Genealogías de la reforma sanitaria (Madrid: Catarata, 2020).

9 Juli Nadal, La construcción de un éxito. Así se hizo nuestra sanidad pública (Barcelona: Ediciones La Lluvia, 2016).

${ }_{10}$ Organización Mundial de la Salud, Alma-Ata 1978. Atención Primaria de Salud (Ginebra: Organización Mundial de la Salud, 1978).
} 


\section{¿Hubo educación sanitaria durante el franquismo?}

Las afirmaciones del preámbulo de Cuadernos para el Diálogo y del texto de Artigas y Pardell a propósito de la ausencia de educación sanitaria en el franquismo, se pueden suscribir si la consideramos tal y como la describió Yuste. Sin embargo, desde un enfoque tradicional ${ }^{11}$ de trasmisión vertical, de arriba abajo, habitual en el último cuarto del siglo XIX y primero del XX - y que no desapareció en los proyectos de reforma sanitaria - sí hubo actividades que trataron de educar a la población para, sobre todo, permitir a los sanitarios desarrollar su labor. Fueron propuestas dispersas, en un contexto en el que se primó la medicina curativa con la puesta en marcha en 1944 del Seguro Obligatorio de Enfermedad (SOE), ${ }^{12}$ postergando la preocupación por la salud de la colectividad. ${ }^{13}$ Conocerlas es preciso para entender el punto de partida de los debates surgidos en la Transición.

\section{LA EDUCACIÓN SANITARIA EN EL PRIMER FRANQUISMO}

Durante esta etapa hubo una cierta continuidad con las iniciativas de educación sanitaria (entonces "propaganda sanitaria») desarrolladas durante la II República. En plena guerra se estableció un Servicio de Divulgación y Propagada Sanitarias, cuyo punto de partida fue la idea de: «[...] que uno de los factores más importantes en la lucha contra las deficiencias biológicas, los estados de inferioridad física, las enfermedades infecciosas y cuanto abarca y trata de combatir la Higiene Pública, es la incultura popular sobre estas materias [...]».14 Por ello se organizaba un «[...] servicio especial encargado de modelar la conciencia sanitaria del público». Lo dirigió el dermatólogo

\footnotetext{
${ }^{11}$ La continuidad de esta perspectiva en el caso de la educación relacionada con el cuidado de la infancia, de gran relevancia durante el franquismo, ha sido estudiada en Irene Palacio Lis, Mujeres ignorantes: madres culpables adoctrinamiento y divulgación maternoinfantil en la primera mitad del siglo XX (València: Universitat de València, 2003).

12 Eduardo Bueno Vergara y Enrique Perdiguero-Gil, «Mejor curar que prevenir: dispositivos asistenciales y actividades preventivas en el primer franquismo», en Del siglo XIX al XXI. Tendencias y debates, eds. Mónica Moreno Seco, Rafael Fernández Sirvent y Rosa Ana Gutiérrez Lloret (Alicante: Biblioteca Virtual Miguel de Cervantes, 2019), 1972-1983.

13 Pedro Marset Campos, José Miguel Sáez Gómez y Fernando Martínez Navarro, «La Salud Pública durante el franquismo», Dynamis 15 (1995): 211-250.
}

${ }^{14}$ Boletín Oficial del Estado de 31 de mayo de 1938, n. ${ }^{\circ}$ 586: 7610-7611. 
Julio Bravo Sanfeliú, que ya había encabezado la sección de Higiene Social y Propaganda de la Dirección General de Sanidad (DGS) creada en $1931 . .^{15}$

A partir de las fuentes consultadas, se puede inferir que el Servicio de Divulgación y Propagada Sanitarias (después Sección de Propaganda de la DGS) no desarrolló una gran actividad. No obstante, confeccionó carteles para las «luchas sanitarias» contra la mortalidad infantil, el paludismo, la polio, las enfermedades venéreas y el cáncer, sobre higiene, los peligros de la circulación, y también para la lotería de la Cruz Roja. También llevó a cabo charlas radiofónicas. ${ }^{16}$

Por su parte, la facción falangista del Régimen, aunque desde las páginas de la revista SER se adhirió a la necesidad de la propaganda sanitaria, ${ }^{17}$ en no pocas ocasiones mostró reticencias ante las actividades divulgativas, constriñéndolas a un estrecho marco: fomentar la inmunización y la profilaxis, recurrir al médico ante cualquier duda, promover revisiones médicas periódicas y prevenir lo que se consideraban abusos en la utilización del SOE. ${ }^{18}$

La DGS publicó, entre 1944 y 1962, una colección de 68 folletos destinados a actualizar los conocimientos de los médicos rurales. ${ }^{19}$ No obstante,

\footnotetext{
${ }_{15}$ Ramón Castejón Bolea, Enrique Perdiguero Gil y José Luis Piqueras Fernandez, eds., Las imágenes de la salud: cartelismo sanitario en España (1910-1950) (Alicante. Madrid: Instituto Alicantino de Cultura «Juan Gil-Albert». Consejo Superior de Investigaciones Científicas, 2012). Ramón Castejón, Enrique Perdiguero y Rosa Ballester, «Los medios de comunicación al servicio de la lucha antivenérea y la protección de la salud maternoinfantil (1900-50)», História, Ciências, Saúde-Manguinhos 13, n. ${ }^{\circ} 2$ (2006): 411-437. Enrique Perdiguero, Rosa Ballester y Ramón Castejón, «Films in Spanish Health Education: The Case of Child Health (1928-1936)», Higyea Internationalis 6, n. 2 (2007): 69-97.
}

16 Julio Bravo, Algunas consideraciones sobre propaganda en general y propaganda sanitaria en particular (Madrid: Dirección General de Sanidad, 1951).

${ }_{17}$ Fue la facción falangista del Régimen la que puso en marcha, desde el Ministerio de Trabajo, el SOE. La revista SER era una publicación de la Delegación de Sanidad de Falange. Enrique Perdiguero-Gil, «Propaganda y "educación sanitaria" en el ideario médico-social del Franquismo a través de la revista SER», en Política, salud y enfermedad en España: entre el desarrollismo y la transición democrática, ed. Enrique Perdiguero-Gil, (Elche: Universidad Miguel Hernández, 2015).

${ }^{18}$ Dr. Turégano, «Un aspecto de la propaganda en el seguro», SER X, n. 90 (1951): 78. Se rechazaba todo elemento que diera a conocer a la población síntomas de las enfermedades y tratamientos de las mismas: Perdiguero-Gil, «Propaganda y "educación sanitaria”».

19 Bravo, Algunas consideraciones sobre propaganda, 22. 
algunos dedicados al cáncer, tuvieron un claro interés divulgativo. ${ }^{20}$ También publicó, entre 1938 y 1964, una colección de monografías, "Al Servicio de España y del Niño Español», inserta en la política pronatalista del Régimen, cuya audiencia fue profesional: los sanitarios implicados en el cuidado de la salud maternoinfantil, dependientes del Ministerio de Gobernación y ajenos a los pediatras del SOE. Sin embargo, alguna monografía también puede encuadrarse en el ámbito de la educación sanitaria de las madres. ${ }^{21}$

Por su parte, las divulgadoras rurales de la Sección Femenina desplegaron actividades de educación sanitaria, tanto por sí mismas como a través de las cátedras ambulantes. Si bien esta institución ha sido profusamente estudiada, no existen muchos análisis sobre las tareas de educación sanitaria. ${ }^{22}$ Sin duda, su actividad, fuertemente adoctrinadora, fue relevante, especialmente en la década de los cuarenta. ${ }^{23}$

Otra figura relacionada con la educación sanitaria, las enfermeras de Salud Pública (su nombre oficial fue variando con el tiempo), a las que se había otorgado un importante papel durante la II República, fue perdiendo relevancia. Varias de las mejor formadas acabaron en el exilio

\footnotetext{
${ }^{20}$ Fueron textos premiados por la Asociación Española Contra el Cáncer, creada en 1953, que estableció un concurso con afán vulgarizador. Por ejemplo: A. Pérez Martínez, Lo que todo el mundo debe saber sobre el cáncer (Madrid: Dirección General de Sanidad, 1954).
}

${ }_{21}$ Modesta Salazar Agulló, Emilio Martínez Marco y Josep Bernabeu-Mestre, «La salud maternoinfantil durante el franquismo: notas bibliométricas sobre el programa "Al servicio de España y del niño español"», Asclepio 59, n. 1 (2007): 285-314.

${ }_{22}$ Heliodoro Manuel Pérez Moreno, «La asistencia sanitaria cono función circumformativa de la Cátedra Ambulante de Huelva (1956-1977)», Cuestiones Pedagógicas, n. 21 (2011/2012): 197-228; Heliodoro Manuel Pérez Moreno, «Educación y asistencia social de una escuela errante durante el Franquismo en España», Revista de Educación Social, n. 17 (2013): 1-16. Alberto González García, «La Sección Femenina y las campañas de vacunación obligatoria contra la difteria en España en las páginas de La Vanguardia (1940-1955)», Vínculos de Historia. Revista del Departamento de Historia de la Universidad de Castilla-La Mancha 5 (2016): 309-329, https://doi.org/10.18239/vdh. v0i5.018. José Manuel Maceiras-Chans, María Eugenia Galiana-Sánchez y Josep Bernabeu-Mestre, «La Sección Femenina en las campañas de vacunación del primer franquismo: el caso de la provincia de Valencia (1941-1958)», RECIEN 13 (2017): 5-23, https://doi.org/10.14198/recien.2017.13.02.

${ }_{23}$ José Manuel Maceiras-Chans, María Eugenia Galiana-Sánchez y Josep Bernabeu-Mestre, «Enfermería y control social: las actividades socio-sanitarias de la Sección Femenina de Falange en la ciudad de Valencia (1940-1977)», Enfermería Global 17, n. 1 (2018): 270-282, https://doi.org/10.6018/ eglobal.17.1.263381. No hay que olvidar el papel que en la educación de las mujeres tuvo el Servicio Social: Beatriz Echeverri Dávila, «La protección de la infancia: la educación sanitaria de las madres en la posguerra española», Historia y Política, n. ${ }^{\circ} 9$ (2003): 279-308. 
y la labor de las que quedaron fue diluyéndose con la reorientación de la enfermería. ${ }^{24}$

En resumen, en las dos décadas iniciales de la Dictadura, las actividades de propaganda y divulgación sanitaria — fueron los términos más usados-, impulsadas desde la sanidad oficial, fueron débiles y dispersas, dedicadas a combatir las creencias de la población, concebidas como un lastre para la actividad sanitaria, especialmente en el caso de la salud maternoinfantil.

\section{LA EDUCACIÓN SANITARIA EN EL DESARROLLISMO}

La educación sanitaria se mantuvo al margen de la estructura asistencial del SOE, que progresó notablemente con la puesta en marcha de la Seguridad Social en 1967. Excluido lo relacionado con el programa EDALNU, cuyo desarrollo se produjo principalmente en el ámbito escolar, ${ }^{25}$ los planteamientos de la educación sanitaria no cambiaron con respecto a los de la etapa anterior, como muestran las palabras de Bosch, jefe de los Servicios de Sanidad Maternoinfantil de la DGS: «Hace falta una mínima cultura sanitaria que en el orden individual y familiar acepte y hasta reclame las prácticas de medicina preventiva y en el orden social acepte también las medidas colectivas, colabore al bienestar de la comunidad y sienta la responsabilidad de medidas sanitarias [...]».26

La frase forma parte de su intervención en la V Conferencia de la Unión Internacional para la Educación Sanitaria de la Población (UIESP), celebrada en Filadelfia en 1962. ${ }^{27}$ La siguiente conferencia, en

\footnotetext{
${ }^{24}$ Josep Bernabeu-Mestre y Encarna Gascón Pérez, Historia de la Enfermería de Salud Pública en España (1860-1977) (Alicante: Publicaciones de la Universidad de Alicante, 1999). Josep Bernabeu-Mestre y María Eugenia Galiana Sánchez, «Enfermería y exilio. El caso de las visitadoras sanitarias», Mètode, n. 61 (2009): 22-22. María Eugenia Galiana Sánchez, María Pilar García Paramio, y Josep Bernabeu Mestre, «La frustración de una profesión: la enfermería de salud pública en la V Reunión de Sanitarios, 1959», Temperamentvm 5, n. 9 (2009).

${ }^{25}$ Eva María Trescastro-López y Silvia Trescastro-López, «La educación en alimentación y nutrición en el medio escolar: el ejemplo del Programa EDALNU», Revista Española de Nutrición Humana y Dietética 17, n. ${ }^{\circ}$ (2013): e84-e90, https://doi.org/10.14306/renhyd.17.2.11.

${ }^{26}$ Juan Bosch Marín, Filosofía de la educación sanitaria sobre la salud de la madre y el niño (Madrid: Dirección General de Sanidad, 1962), 30.

${ }_{27}$ España se adhirió en 1954 a esta organización, creada en 1951: Marie-Claude Lamarre, «The International Union for Health Promotion and Education», Health Education Research 15, n. ${ }^{\circ}$ (2000): 243-248, https://doi.org/10.1093/her/15.3.243.
} 
1965, tuvo lugar en Madrid. Con tal motivo se publicó el libro Problemas de educación sanitaria, ${ }^{28}$ en el que participaron autores como Adolfo Maíllo y Primitivo de la Quintana quienes, desde el mundo educativo y sanitario, ya habían mostrado su preocupación por la educación sanitaria. ${ }^{29}$ Pero ni el texto de 1962, ni el de 1965, al igual que había ocurrido con los previos relacionados con la UIESP de 1955 y 1959,30 indican que la educación sanitaria en España hubiera traspasado el dintel de los esfuerzos débiles y descoordinados ya comentados. ${ }^{31}$ De hecho, en 1962 fracasó un intento de poner en marcha, con el asesoramiento de la OMS, un departamento de educación sanitaria en el seno de la Escuela Nacional de Sanidad. ${ }^{32}$

En lo programático, sí se ofrecieron novedades respecto al planteamiento más clásico de la labor educadora como lucha contra la ignorancia. Tal es el caso de Adolfo Serigó, muy activo en diversos frentes sanitarios durante el franquismo. ${ }^{33}$ En una obra dedicada a la asistencia a los enfermos mentales, incluyó un capítulo sobre educación sanitaria en el que, muy atento a las discusiones internacionales sobre el tema, abogó por considerar las bases culturales de la salud y la enfermedad, de modo que con anterioridad a cualquier actividad educativa debía

\footnotetext{
28 V.V. A.A., Problemas de educación sanitaria. Curso desarrollado como contribución a la VI Conferencia de la Unión Internacional de Educación Sanitaria (Madrid: Editorial Universitaria Europea, 1965).

${ }_{29}$ Véase los trabajos al respecto ya citados en el artículo de Terrón y Hurtado en este mismo monográfico.

30 Unión Internacional para la Educación Sanitaria de la Población (Madrid: Dirección General de Sanidad, 1955), 39-42. Manuel Blanco Otero y Enrique Bravo, Unión Internacional para la Educación Sanitaria Popular (IV Conferencia, Düsseldorf, 2-9 mayo 1959) (Madrid: Dirección General de Sanidad, 1959), 33-37.

${ }^{31}$ No hay estudios detallados sobre otras vías de educación sanitaria tanto institucionales como no institucionales, en especial en el ámbito audiovisual. A modo de ejemplo podemos citar el programa radiofónico «El consejo del doctor» emitido por la Sociedad Española de Radiodifusión entre 1962 y 1974. Lo dirigía el yerno de Franco, Cristóbal Martínez-Bordiú, controvertido cirujano. En los primeros años contó con guiones teatrales a cargo del periodista Antonio Domínguez Olano. Posteriormente adoptó un formato periodístico. Todos los meses ofrecía un premio a las contribuciones de médicos españoles sobre la importancia de la divulgación de la salud.

32 Pilar Nájera, «Health Education in Spain - AN OVERVIEW», en World Yearbook of Educacion. Health Education, eds. Chris James, John Balding, y Duncan Harris (London: Kogan Page, 1989), 64-72.

33 Enric J. Novella, «Los límites de la tecnocracia: el Patronato Nacional de Asistencia Psiquiátrica y la modernización autoritaria de la asistencia psiquiátrica en la España del segundo franquismo», Dynamis 39, nº 1 (2019): 73-97, https://doi.org/10.30827/dynamis.v39i1.8667.
} 
hacerse un análisis cultural de la población en la que se iba a intervenir. ${ }^{34}$ Las ideas de Serigó no tuvieron efecto práctico.

Unos años antes, el completo informe del Prof. Colin F. Brockington, ${ }^{35}$ uno de los consultores enviados por la OMS en el marco de la colaboración entre el organismo internacional y España, puso de manifiesto la mala situación de la administración sanitaria española en general y, por tanto, de la educación sanitaria en particular. Subrayó la inexistencia de la enfermería de Salud Pública y la falta de formación especializada.

El informe Brockington hay que contextualizarlo en el ámbito del reformismo sanitario franquista. En 1965, se había confeccionado un ambicioso Plan de Actividades Sanitarias, sin traducción práctica, ${ }^{36}$ que incluía un apartado sobre la educación sanitaria, estableciendo la labor directiva de la DGS en lo referido a la materia. ${ }^{37}$ Planteaba acciones a varios niveles, muchas de ellas novedosas, si bien el punto de partida era tradicional: «No parece preciso extenderse muy ampliamente en demostrar la necesidad de modificar la actitud de indiferencia de la mayor parte de la población en cuanto a los problemas sanitarios en general, en el aspecto privado como en el colectivo». ${ }^{38}$

También se planteó poner en marcha zonas de demostración sanitaria, en las que llevar a cabo la integración de todas las actividades sanitarias, incluida la educativa. Se planificó una en Talavera de la Reina, pero no se inauguró hasta $1976 .{ }^{39} \mathrm{El}$ proyecto de Talavera fue una de las

\footnotetext{
${ }^{34}$ Adolfo Serigó Segarra, «Las bases de la educación sanitaria», en Bases sociales de la asistencia a los enfermos mentales (Madrid: Patronato Nacional de Asistencia Psiquiátrica, 1969), 282-299. Serigó era el secretario del Plan Nacional de Asistencia Psiquiátrica.

${ }_{35}$ Esteban Rodriguez-Ocaña, «La sanidad franquista vista desde la Organizacion Mundial de la Salud: el Informe Brockington (1967)», Gaceta Sanitaria 32, n. 6 (2018): 582-583, https://doi.org/10.1016/j.gaceta.2018.03.004.

${ }^{36}$ Rodríguez Ocaña y Ballester Añón, «El Informe del consultor de la OMS Fraser Brockington», 480-484.

37 Plan de Actividades Sanitarias (Madrid: Dirección General de Sanidad), 78-83. El apartado dedicado a la educación sanitaria mantenía la denominación tradicional: «Propaganda y Divulgación Sanitaria dirigida a la población».

38 Plan de Actividades Sanitarias, 78.

${ }_{39}$ Esteban Rodríguez Ocaña y Juan Atenza Fernández, «El proyecto E30 OMS-España para el establecimiento de una zona de demostraciones sanitarias en Talavera de la Reina, 1965-1976», en Salud, enfermedad y medicina en el franquismo, eds. $\mathrm{M}^{\mathrm{a}}$ Isabel Porras Gallo, Lourdes Mariño Gutiérrez, y María Victoria Caballero Martínez (Madrid: Catarata, 2019), 124-145.
} 
escasas referencias a la educación sanitaria en los Planes de Desarrollo Económico y Social. ${ }^{40}$

La afirmación sobre la «indiferencia» a la que se aludía en el Plan de Actividades Sanitarias contrasta con el surgimiento durante el desarrollismo de un pujante mercado editorial sobre popularización médica, en especial «guías médicas para el hogar», que daban consejos preventivos, para entender las enfermedades y realizar primeros auxilios. ${ }^{41}$ Era un tipo de vulgarización repudiada por muchos médicos, entre los que también se contaban algunos de los que propusieron reformas. ${ }^{42}$

En julio de 1975, pocos meses antes de la muerte de Franco, una comisión interministerial creada para abordar la reforma sanitaria, emitió un informe en el que la educación sanitaria se situaba en coordenadas más próximas a las formuladas por Yuste: «La educación sanitaria de la población y el fomento de la solidaridad constituyen aspectos fundamentales de profunda raíz social y comunitaria». ${ }^{43}$

También en julio 1975 se puso en marcha tanto el departamento de Educación Sanitaria de la Escuela Nacional de Sanidad, ${ }^{44}$ encabezado por Pilar Nájera (1930-2018), ${ }^{45}$ como la sección de Educación Sanitaria de la DGS que tuvo continuidad en el Ministerio de Sanidad y Consumo. ${ }^{46}$ Pilar Nájera era en 1975 la única mujer en el escalafón del Cuerpo de Médicos de Sanidad Nacional y había dedicado varios años a la

\footnotetext{
40 Perdiguero-Gil y Comelles, «The defence of health»: 52-54.

${ }^{41}$ Enrique Perdiguero Gil, «La popularización médica durante el franquismo: un primer acercamiento», en Educación, comunicación salud. Perspectivas desde las ciencias humanas y sociales, coords. Josep M Comelles y Enrique Perdiguero Gil, (Tarragona: Publicacions Univeristat Rovira i Virgili, 2017), 105-138.

42 José Antonio Valtueña, «El ciudadano indefenso y la educación para la salud», Cuadernos para el Diálogo Extra XLVI (1976): 77-78.

${ }_{43}$ Comisión Interministerial para la Reforma Sanitaria, Informe al Gobierno, (Madrid: Ministerio de la Gobernación. Comisión Interministerial para la Reforma Sanitaria, 1975), 9.

${ }^{44}$ Nájera, «Health Education in Spain - AN OVERVIEW», 65.

${ }^{45}$ En el Boletín Oficial del Estado de 8 de febrero de 1974, n. ${ }^{\circ}$ 34: 2483 se publicó el nombramiento de María Pilar Nájera Morrondo como Jefe del Departamento de Educación Sanitaria de la Escuela Nacional de Sanidad.

46 En el Boletín Oficial del Estado de 16 de julio de 1982, n. ${ }^{\circ}$ 169: 19303 se publicó el nombramiento de Pilar Nájera Morrondo como Jefe de la Sección de Educación Sanitaria del Ministerio de Sanidad y Consumo.
} 
gestión hospitalaria antes de pasar a ser el referente en la sanidad oficial de la educación sanitaria. Sobre las actividades realizadas por el departamento y la sección en sus primeros años de funcionamiento, los que nos incumben aquí, no hay demasiadas noticias, más allá de la intensa labor docente realizada, sobre todo en el tema de la educación nutricional, en estrecha colaboración con el ya citado programa EDALNU. ${ }^{47}$ Las publicaciones más sobresalientes de Pilar Nájera más allá de un texto para manipuladores de alimentos, editado varias veces, se produjeron a finales de los ochenta y noventa del pasado siglo, ya en el contexto de la Educación para la Salud, esquema conceptual predominante en aquellos años. La labor de Pilar Nájera, sin embargo, no fue reconocida por los implicados en la reforma sanitaria, probablemente porque su planteamiento, más técnico, estaba alejado del papel emancipador que se quiso otorgar a la educación sanitaria.

\section{La educación sanitaria en la reforma sanitaria}

La importancia de la educación sanitaria en el contexto de la reforma aparece, sobre todo, en los proyectos que se publicaron por parte de variados agentes sociales (médicos, sociólogos, economistas, periodistas y miembros de movimientos sociales), que podemos denominar de manera genérica como «oposición democrática», antes y después de la legalización de los partidos políticos. Fueron los miembros del Partit Socialista Unificat de Catalunya (PSUC) y del Partido Comunista de España (PCE) los que se mostraron más activos a la hora de proponer un nuevo sistema sanitario. ${ }^{48}$ En general, estos escritos tomaron como referencia sistemas sanitarios europeos, particularmente los de Reino Unido e Italia.

\footnotetext{
${ }_{47}$ María Teresa García Jiménez, «Introducción. Breve historia de la alimentación y la nutrición en los colegios españoles. Recursos y currículo», Nutrición y Alimentaciónn en el ámbito escolar, en ed. Jesús Román Martínez Álvarez, (Majadahonda, Madrid: Ergon, 2012), 13-14.

${ }_{48}$ Muchos de ellos habían participado en el ya citado Xè Congrés de Metges i Biòlegs en Llengua Catalana de 1976. PSUC y PCE, además, celebraron jornadas sanitarias en aquellos años: Partit Socialista Unificat de Catalunya, I Jornades de Sanitat del PSUC. Ponències i comunicacions. 11-12 de febrer de 1978 (Barcelona: Comissió de Sanitat del Comité Central. PSUC, 1978). Partido Comunista de España, Primeras Jornadas Sanitarias del PCE. Madrid. 10 y 11 de Junio, 1978. Ponencias y Comunicaciones (Madrid: Partido Comunista de España, 1978). También en junio de 1978 el PSOE organizó unas Jornadas Federales de Salud Pública, cuyas propuestas, al parecer, no diferían mucho de las anteriores: Jesús M. de Miguel Rodríguez, La sociedad enferma: las bases sociales de la política sanitaria española (Madrid: Akal, 1979), 191; "Propuesta de creación de un "Servicio Nacional de la Salud"», El País, junio 27, 1978.
} 
En este último caso, hay que tener en cuenta que los médicos socialistas y comunistas desempeñaron un papel preeminente en las discusiones sobre la reforma sanitaria italiana de 1978, destacando la gran influencia de los trabajos de Bassaglia, en el campo psiquiátrico, y de Seppilli y Berlinguer, en salud pública y políticas sanitarias. ${ }^{49}$ También tuvieron influencia las experiencias de algunos países latinoamericanos. ${ }^{50}$

Todas las propuestas de quienes se identificaron con la «oposición democrática» tenían varios rasgos comunes, si bien hubo numerosos matices - algunos de ellos solo estaban referidos a Cataluña-. ${ }^{51} \mathrm{El}$ modelo preferido fue un servicio nacional de salud con cobertura universal para la población española financiado con cargo al presupuesto del Estado. Para conseguir este objetivo, España necesitaba un régimen fiscal moderno (introducido en 1977) 52 y, por tanto, también era necesario diseñar una etapa transitoria en la financiación del sistema sanitario.

Otra característica esencial del servicio de salud propuesto fue la prestación de servicios integrales: educación sanitaria, salud pública y

${ }_{49}$ V.V. A.A., Medicina y sociedad (Barcelona: Fontanella, 1972). Jesús M. de Miguel Rodríguez, «Policies and politics on the health reforms in Southern European Countries: a sociological critique», Social Science and Medicine 11 (1977): 379-393. Giovanna Vicarelli, «The creation of the National Health System in Italy (1961-1978)», Dynamis 39, n. ${ }^{\circ}$ (2019): 21-43, https://doi.org/10.30827/dynamis.v39i1.8665.

50 Juan Carlos Duro Martínez, «Discursos médicos y políticos sobre la salud comunitaria durante la transición democrática española», Praxis Sociológica, n. ${ }^{\circ} 18$ (2014): 56.

${ }^{51}$ Estas propuestas fueron publicadas en diversas obras, muchas de ellas colectivas, que recogían, en su mayor parte, ideas ya discutidas en el Congrés de 1976. Entre las más significativas están Alberto Infante, ed., Cambio social y crisis sanitaria. (Bases para una alternativa) (Madrid: Ayuso, 1975). Nolasc Acarín et al., La sanidad hoy. Apuntes críticos y una alternativa (Barcelona: Avance, 1975). Nolasc Acarín et al., La salud, exigencia popular (Barcelona: Laia, 1976). Nolasc Acarín et al., Servicio Nacional de Salud. Una alternativa democrática a la sanidad (Barcelona: Laia, 1977), que recoge, básicamente, las mismas ideas del Congrés de 1976 de Perpiñán. Jordi Gol i Gurina et al., La sanitat als Països Catalans. Crítica i Documentació (Barcelona: Edicions 62, 1978), 1-96; Carlos Borasteros, Salud, enfermedad y sociedad (Madrid: Forma, 1978). Para un análisis detallado de estos proyectos y de las referencias bibliográficas concretas de los puntos discutidos remitimos a Perdiguero-Gil y Comelles, «The defence of health». Jesús M. de Miguel publicó varios análisis de las propuestas de los partidos políticos de la oposición en los mismos años en los que se estaban produciendo. El más acabado se recoge en: de Miguel, La sociedad enferma. De Miguel critica ferozmente los modelos sanitarios propuestos por la oposición. Por ejemplo, los debates en torno al concepto de salud los consideró «[...] parte de un replanteamiento epistemológico tradicional de toda la sanidad, que convendría superar cuanto antes...» (p. 188).

52 Juan Pan-Montojo, «Entre la reforma y las contrarreformas: una historia política de la fiscalidad española en la democracia», en eds. Carlos Barciela López, Antonio Di Vittorio y Joaquín Melgarejo Moreno, La evolución de la Hacienda Pública en Italia y España (siglos XVIII-XXI) (Alicante: Publicacions de la Universitat d'Alacant, 2015), 557-590. 
medicina preventiva, atención sanitaria, rehabilitación y reintegración social. La defensa de la salud, en lugar de la curación, sería el principal objetivo. Para priorizar la prevención de enfermedades era fundamental identificar sus causas biológicas, ambientales y sociales. En este contexto, se consideró indispensable la participación de la población en la planificación, gestión y evaluación de los servicios de salud. Este elemento fue subrayado en las propuestas comunistas que se inspiraron en las del Partido Comunista Italiano, que descansaban en el importante papel atribuido a los municipios y barrios en el desarrollo de las actividades sanitarias.

Otro objetivo de la reforma sanitaria fue superar las desigualdades de acceso a la asistencia, mediante la «regionalización» y la «descentralización». La idea era organizar todas las actividades de salud a través de una red jerárquica de centros con diversos grados de especialización. Si bien todas las propuestas que hemos analizado comparten estos rasgos generales, la mayoría hace hincapié en la última cuestión. La paradoja de detallar lo curativo en el contexto de un modelo integral se debe a que muchos de los promotores de la reforma sanitaria eran médicos con actividad hospitalaria.

La mayoría de los proyectos incidieron reiteradamente en los mismos temas, sin demasiada concreción y sin una valoración económica de lo que se proponía. Los escritos más originales fueron los que exploraron con cierto detalle las consecuencias de establecer la defensa de la salud y, por tanto, la educación sanitaria, como piedra angular de los servicios de salud. Surgieron varios enfoques, generalmente superpuestos: la conceptualización de la salud, el sesgo biológico de la medicina científica, la necesidad de incorporar la idea de multicausalidad y, por tanto, considerar el papel de la estructura socioeconómica en la génesis de las enfermedades.

La discusión más influyente y amplia sobre el concepto de salud la ofreció el médico generalista catalán Jordi Gol (1924-1985). Criticó los conceptos imperantes, especialmente el de la OMS, ${ }^{53}$ y propuso una noción dinámica basada en la capacidad de alcanzar la autorrealización a

\footnotetext{
53 «[...] a state of complete physical, mental and social wellbeing [...]». World Health Organization,
} Official Records of the World Health Organization, n. 2 (Geneva: World Health Organization, 1948), 16. 
través de la autonomía, la solidaridad y la felicidad. Los médicos del PCE respaldaron el concepto propuesto por el salubrista Enrique Nájera (1934-1994), hermano de la ya citada Pilar Nájera, quien destacó el equilibrio social y la integración en la sociedad. Ambos autores destacaron el valor positivo de la salud y la relación dinámica con factores biológicos, ambientales y sociales.

Por tanto, el objetivo de los servicios de salud debía ser evitar cualquier circunstancia que obstaculizase la realización personal, la integración social y la felicidad, mediante la redistribución de la riqueza para evitar desigualdades por clase social o distribución geográfica. Así, las actividades sanitarias, más que en la curación, debían concentrarse en los procesos que generaban enfermedad. La educación sanitaria se incardinaba, por tanto, en este afán de reorientación de los servicios sanitarios, que requería más «ruptura» que «reforma».54 Guiados por un conocimiento médico científico no solo basado en lo biológico, serían parte de una estrategia global que condujese a cambios sociales de base. La consideración de las causas sociales de las enfermedades y la participación de la población en la gestión de los servicios de salud, expondrían las contradicciones del sistema capitalista y fomentaría movilizaciones y/o iniciativas «revolucionarias». Por tanto, las propuestas de algunas fuerzas políticas de izquierda en torno a la educación sanitaria, especialmente las del PSUC y el PCE, se asociaron con una estrategia política general en clave revolucionaria.

Estrechamente relacionada con el problema del sesgo biologicista, tan patente en el desarrollo del conocimiento médico, estaba la cuestión de cómo y qué se debía enseñar en la formación médica, ya que los nuevos servicios de salud requerían un tipo diferente de profesional. ${ }^{55} \mathrm{Se}$ propusieron algunas soluciones, pero, como ocurrió en otros muchos asuntos, no se consideró con detalle un nuevo programa docente. Poco se dijo sobre la formación sanitaria que permitiese el estudio de los

\footnotetext{
${ }^{54}$ Los proyectos de la oposición trataron de huir del término «reforma sanitaria» ya utilizado por las autoridades en el tardofranquismo y por los gobiernos de la Unión del Centro Democrático.

55 Más de veinte años después se volvía a problemas ya explorados y no solventados: Primitivo de la Quintana, «La medicina social como problema docente (I)», Revista de Educación 5, n. 12 (1953): 1-11. Primitivo de la Quintana, "La medicina social como problema docente (II)», Revista de Educación 5, n. 13 (1953): 110-128. Veáse el artículo de Bacerló-Prats i Comelles en este monográfico.
} 
determinantes socioeconómicos y políticos de las enfermedades y el desarrollo de actividades preventivas y de educación sanitaria.

Por tanto, en el contexto de los nuevos conceptos de salud y servicios de salud propuestos por los reformadores o rupturistas, el papel de la educación sanitaria fue considerado crucial. Pero ¿qué significó «educación sanitaria»?

\section{¿Qué educación sanitaria para un nuevo sistema sanitario?}

La falta de reconocimiento de la complejidad de la educación sanitaria de la población — a la que nos hemos referido al inicio de este textoha de enmarcarse en las muy abundantes pero poco concretas referencias a la misma que se hicieron en los escritos sobre la reforma sanitaria, tanto desde el gobierno como desde la oposición. En los proyectos de esta última, aparecieron de manera reiterada propuestas estereotipadas en las que la educación sanitaria formaba parte de un modelo integral: «[...] el Servicio Nacional de la Salud, implica cubrir sanitariamente toda la población e integrar, homogeneizar y unificar todos los servicios sanitarios en un conjunto armónico que incluye la educación sanitaria de la población, la higiene pública y epidemiología, la prevención, la curación y la rehabilitación y reinserción social».56 La educación sanitaria no era un elemento más, era la base de la pirámide del sistema sanitario, pues la prevención podía evitar la enfermedad y por tanto la necesidad de curar. En algunas ocasiones se subrayó la rentabilidad de este enfoque, al evitar los elevados costes de lo asistencial. ${ }^{57}$ El escenario privilegiado para desarrollar la educación sanitaria era la Atención Primaria de Salud, canonizada en Alma-Ata. En algunos casos se dedicaron algunas líneas algo más específicas:

La educación sanitaria. Pilar fundamental de los servicios de salud, trata de dar a la población la información necesaria sobre cuáles son los hábitos de vida más saludables, esclarecer sobre las costumbres sanas o insanas, dar la formación necesaria para el conocimiento del conjunto psicofísico personal y de las influencias

\footnotetext{
${ }^{56}$ Acarín et al., Servicio Nacional de Salud, 48.

${ }^{57}$ Adolfo Serigó Segarra, La crisis sanitaria en España (Nuevas ideas en salud pública) (Madrid: El autor, 1979), 60.
} 
externas; orientar sobre el uso de medicamentos y tóxicos. Poniendo especial atención a determinados grupos sociales: niños, adolescentes, madres, ancianos, etc.

Todo esto desarrollado por personal especializado en educación sanitaria, que podrá ser reclutado entre los profesionales sanitarios de hoy, partiendo de una formación específica. ${ }^{58}$

Era una visión nada novedosa de la acción educativa en torno a la salud, criticada pocos años después por la OMS: «Muchos administradores siguen creyendo que ver una película, contemplar un cartel o escuchar una conferencia conducirán al individuo por el buen camino»,59 y por el texto de 1987 ya referido: "No se trata tanto de saber elaborar consignas salutíferas (con pretensión más o menos moralizante $[\ldots] » .60$

Sin mayor concreción, pero con una visión menos vertical, hubo otras formulaciones:

Així l'educació sanitària pot ser quelcom mes que consells tècnics que el sanitari dona a la població, i convertir-se en un procés d’informació i aportació reciproca, on el ciutadà rep unes informacions que pot contrastar amb la seva vida diària, i el sanitari obté uns coneixements del medi on treballa i ensems pot refer aspectes del seu saber una vegada confrontats i discutits amb la població. ${ }^{61}$

Con frecuencia, la educación sanitaria fue un elemento primordial en la construcción de un nuevo sistema sanitario que suponía cambios políticos: "L'acció educativa esta dirigida especialment a aconseguir la participació de la població en la planificació i gestió de l’acció sanitària,

\footnotetext{
58 Acarín et al., Servicio Nacional de Salud, 53.

59 Organización Mundial de la Salud, Nuevos métodos de educación sanitaria en la atención primaria de salud. Serie Informes Técnicos, 690 (Ginebra: Organización Mundial de la Salud, 1983), 11.

${ }^{60}$ Centre d'Anàlisi i Programes Sanitaris, «Nota editorial», 5.

61 Nolasc Acarín, «Bases organizativas per a la defensa de la salut», en Xè Congrès de Metges $i$ Biòlegs de Llengua Catalana. II Ponència. Funció social de la medicina, (Barcelona: Acadèmia de Ciències Mèdiques de Catalunya i de Balears. Societat Catalana de Biologia, 1976), 303. Se trata de una visión más cercana a: Organización Mundial de la Salud, Nuevos métodos de educación sanitaria en la atención primaria de salud. Serie Informes Técnicos, 690.
} 
de manera que aquesta responga a les necessitats reals de la població».62 A pesar de que para conseguir tal cosa se definieron diversos niveles e instrumentos de participación, tampoco fueron propuestas detalladas.

La falta de concreción en lo referido a la educación sanitaria sería congruente con la apreciación del sociólogo Jesús M. de Miguel, sobre el conjunto de las propuestas de reforma sanitaria: «Faltan, sin duda, en España modelos alternativos al sistema sanitario actual con suficiente elaboración como para poder aplicarse con soltura. El nivel de discusión de la oposición en este terreno es todavía muy básico y en cierta forma atrasado $[\ldots] » .^{63}$

Tampoco fueron más detalladas las propuestas gubernamentales. Tras los fallidos intentos de Enrique Sánchez de León, ${ }^{64}$ primer ministro de Sanidad de la Transición ${ }^{65}$, en mayo de 1980, fue llevado a las Cortes un proyecto de reforma sanitaria:

Se entiende así la educación sanitaria como la interrelación entre los servicios sanitarios y la población que hace uso de ellos. Determina que se utilizarán sistemas permanentes de formación sanitaria y modernos métodos de transmisión de la misma. Se reconoce el empleo de pautas de educación colectiva y de programas de educación sanitaria realizados a través de los Centros de salud. Se señala la ejecución de otros programas a través de empresas, colegios o barrios, en tarea en donde participarán todos los profesionales sanitarios. ${ }^{66}$

En algunas propuestas de la oposición, aunque se reconocía el papel de la escuela en la educación sanitaria, se consideró:

\footnotetext{
${ }^{62}$ Ferran Martínez Navarro, Estructura i malatia. Una alternativas sanitària per al País Valencià (València: Tres i Quatre, 1978), 76.

63 De Miguel, La sociedad enferma, 187.

${ }^{64}$ Véase Manuel Evangelista Benítez, Medicina y sociedad: la reforma sanitaria (Madrid: Ministerio de Trabajo y Seguridad Social, 1981), 329-359.

65 En los gobiernos de Adolfo Suarez, entre 1977 y 1982 hubo cinco ministros de Sanidad. Rovira Tarazona, sustituto de Sánchez de León, encargó el proyecto al secretario de estado de Sanidad, José María Segovia de Arana, prestigioso en el ámbito clínico y académico.

${ }^{66}$ Luis Sánchez Harguindey, Reforma sanitaria española (Madrid: Acción Social Empresarial, 1981 ), 21.
} 
Por encima de ninguna apreciación de casta, el más capacitado para la educación sanitaria es el médico. La consulta médica, en la intimidad de la relación médico enfermo es una labor de educación persona a persona. El médico es el único que tiene los conocimientos científicos básicos o especializados para suministrar guías de acción seguras. ${ }^{67}$

En ocasiones, se aludió a la necesidad de formación complementaria o al cambio de los planes de los estudios médicos. ${ }^{68}$ Se citó al personal de enfermería como susceptible de hacerse cargo de las tareas educativas, pero en este punto, como en otros, el lastre de unos proyectos escritos por médicos fue palmario.

Uno de los puntos que apareció en casi todas las propuestas, fueran del signo que fueran, tiene que ver el papel de la educación sanitaria como antídoto frente a lo que se consideraba uso distorsionado de los servicios sanitarios, bien fuese por infrautilización o, sobre todo, por abuso: «[...] el origen principal de los que acuden al médico y no están realmente enfermos reside en la falta de cultura. Los déficits de información y la ausencia de una educación sanitaria mínima hacen que al menor problema se acuda al médico». ${ }^{69}$ Similar punto de vista aparecía en 1979 en el informe del secretario de estado sobre la reforma sanitaria: «[...] el abuso en la demanda de asistencia médica de la población por la escasez o ausencia de una auténtica educación sanitaria, son también señales claras de la insuficiencia funcional de nuestra estructura sanitaria». ${ }^{70}$

Este punto de vista, especialmente en lo que a las prestaciones farmacéuticas se refiere, que apareció una y otra vez en los primeros años de debate sobre la reforma sanitaria, había sido común tras la puesta en

\footnotetext{
${ }^{67}$ Francisco Javier Yuste Grijalba, Hacia una sociología de la medicina (Barcelona: Aula de Ediciones, 1971), 33.

${ }^{68}$ Nolasc Acarín, «Bases para la regionalización sanitara», en eds. Nolasc Acarín et al., La sanidad hoy. Apuntes críticos y una alternativa, (Barcelona: Avance, 1975), 111-133. Joan Campos i Avillar, "Hacia un modelo de ruptura educativa para la reforma sanitaria», en Planificación y reforma sanitaria, ed. Jesús M. de Miguel Rodríguez (Madrid: Centro de Investigaciones Sociológicas, 1978), $121-151$.

${ }^{69}$ Acarín et al., La salud, exigencia popular, 48.

70 José María Segovia de Arana, «Líneas generales de la reforma sanitaria», junio de 1979, 1137/11, Archivo INGESA, 6.
} 
marcha del SOE ${ }^{71}$, así como en una de las primeras críticas publicadas a la sanidad franquista. ${ }^{72}$ También se señaló el papel de los médicos y de la industria farmacéutica en lo que se consideraba un mal uso de los medicamentos. ${ }^{73}$ La educación sobre el uso de los servicios sanitarios tiene, desde luego, valor, pues permite la optimización de recursos. La cuestión es que tal educación se planteó siempre desde el criterio profesional, sin tener en cuenta las necesidades de la población. En el sentido opuesto, en otras ocasiones se asignó a la educación sanitaria un valor emancipador: el mejor conocimiento de todo lo relacionado con la salud y la enfermedad permitiría al individuo y a las comunidades actuar de forma más independiente frente al paternalismo y hegemonía de los profesionales sanitarios. ${ }^{74}$

Sí hubo, en el contexto de los proyectos reformistas, algunos textos referidos específicamente a la educación sanitaria (o para la salud). José Antonio Valtueña publicó uno muy breve en el número extra en mayo de 1975 de Cuadernos para el Diálogo dedicado a "El derecho a la salud»,75 y Joaquim Jubert publicó dos, similares, en 1976 y $1977 .{ }^{76}$ El Congrés de

\footnotetext{
71 José Fernández Turégano, Comentarios sobre el Seguro de Enfermedad (Madrid: Instituto Provincial de Sanidad. Publicaciones de la Jefatura Provincial de Sanidad de Madrid, 1958), 31-32. Sobre la falta de sostenibilidad del SOE, en parte por el aumento del gasto farmacéutico, véase: Margarita Vilar-Rodríguez y Jerònia Pons-Pons, «The Introduction of Sickness Insurance in Spain in the First Decades of the Franco Dictatorship (1939-1962)", Social History of Medicine 26, n. 2 (2012): 267287, https://doi.org/10.1093/shm/hks082. Para las recomendaciones oficiales sobre el uso de los medicamentos véase: Federico Mayor Domingo, ed., Investigación sobre la asistencia farmacéutica en España: estudio socioeconómico sobre el conjunto de la asistencia sanitaria española (Madrid: Ministerio de Trabajo. Instituto Nacional de Previsión, 1977), 919-921.
}

72 Felipe Solé Sabarís, en, La crisis de la medicina liberal, Henri Hatzfeld (Barcelona: Ariel, 1965), 207.

73 Joaquim Bonal, «Farmàcia e industria farmacèutica», en Xè Congrés de Metges i Biòlegs de Llengua Catalana. II Ponència. Funció social de la medicina, (Barcelona: Acadèmia de Ciències Mèdiques de Catalunya i de Balears. Societat Catalana de Biologia, 1976), 384-398.

${ }^{74}$ Gol i Gurina et al., La sanitat als Països Catalans. Crítica i Documentació, 83.

75 Valtueña, «El ciudadano indefenso», 77-78. Valtueña, médico, continuó interesándose por la educación sanitaria a lo largo de los años. Publicó numerosos artículos sobre diversos temas sanitarios, firmados, como presidente del Centro Internacional de Educación para la Salud (Ginebra, Suiza), organismo sobre el que no hemos encontrado información y, posteriormente, como miembro de la Asociación de Funcionarios Internacionales Españoles. Véase: Perdiguero Gil, «La popularización médica durante el franquismo», 115.

76 Joaquim Jubert i Gruart, «Educació sanitària: pressupòsits bàsics per una pedagogia de la salut», en Xè Congrés de Metges i Biòlegs de Llengua Catalana. II Ponència. Funció social de la medicina, (Barcelona: Acadèmia de Ciències Mèdiques de Catalunya i de Balears. Societat Catalana de Biologia, 1976), 343-353. Joaquim Jubert Gruart, «Educació per la salut. Objectius i etapes», en Salut, 
Cultura Catalana tuvo una comisión dedicada a la educación sanitaria que publicó parte de sus conclusiones. ${ }^{77}$ Varios escritos se ocuparon de la educación sanitaria en el marco más amplio de la sanidad en la escuela. Sus autores fueron médicos como Martí, ${ }^{78}$ Antó, ${ }^{79}$ Casares, y la trabajadora social Francina Roca. ${ }^{80}$

Dejando al margen a Valtueña, quien sí tenía experiencia en actividades de educación sanitaria, una primera cuestión evidente es que, en este conjunto de propuestas más específicas, no hubo participación de autores procedentes del mundo de la pedagogía, algo que sí había ocurrido en el caso de la obra Problemas de educación sanitaria (1965), y en el monográfico de «Vida Escolar» sobre educación sanitaria en la escuela publicado en 1961, aunque solo fuese a través de la presencia, no menor, de Adolfo Maíllo. ${ }^{81}$ Jubert sí estuvo implicado posteriormente en el mundo pedagógico con participación asidua en movimientos de reforma como las escuelas de verano. La inexperiencia de la mayoría de los

sanitat i societat. Per una resposta socialisata a l'actual situació sanitària, eds. Jordi Gol i Gurina et al. (Barcelona: 7 x 7 ediciones, 1977), 59-73. Jubert, neurólogo, interesado en la neurobiología, trabajó en el ámbito de la psicopedagogía y la educación especial. Sobre Jubert véase: Josep María García i Balda, «Aproximació històrica a l'educació especial a Catalunya: l'educació institucionalitzada en centres d'educació especial a les comarques de Girona (1873-1997)»(Tesis Doctoral, Universitat de Girona, 2007).

77 «Comissió d’Educació Sanitaria», en ed. Congrés de Cultura Catalana, Resolucions II, (Barcelona: 1977), 86-89.

78 Josep Martí i Valls, «La sanitat i l'escola», en Xé Congrés de Metges i Biollegs de Llengua Catalana. II Ponència. Funció social de la medicina, (Barcelona: Acadèmia de Ciències Mèdiques de Catalunya i de Balears. Societat Catalana de Biologia, 1976), 361-364. Martí i Valls trabajó en el l'Hospital Universitari Vall d'Hebron, tuvo responsabilidades políticas en el Ajuntament de Barcelona, fundador del CAPS, en los últimos años ha tenido notoriedad en los movimientos de defensa de la sanidad pública en Cataluña.

79 Josep Maria Antó y Josep Martí, "Algunas posibilidades de actuación sanitaria en la escuela», Cuadernos de Pedagogía, n. ${ }^{\circ}$ 31-32 (1977): 64-69. Josep $\mathrm{M}^{\mathrm{a}}$ Antó es epidemiólogo, trabaja actualmente en el Instituto de Salud Global de Barcelona. Véase: «ISGLOBAL. Instituto de Salud Global», 2018. https://www.isglobal.org/our-team/-/profiles/6201, (consultado marzo 15, 2021).

${ }_{80}$ Ramon Casares y Francina Roca, «La medicina i la higiene escolar», en Salut, sanitat $i$ societat. Per una resposta socialista a l'actual situació sanitària, eds. Jordi Gol i Gurina et al. (Barcelona: 7 x 7 edicions, 1977), 155-175. Años después, ambos, junto con otros autores, presentaron un trabajo sobre los profesionales de la salud en el Tretzé Congrés de Metges i Biólegs de Llengua Catalana (Andorra, 1988), en el que hablaron de educación para la salud con algo más de detalle.

81 Adolfo Maíllo, «La educación sanitaria en la escuela», en ed. V.V. A.A., Problemas de educación sanitaria. Curso desarrollado como contribución a la VI Conferencia de la Unión Internacional de Educación Sanitaria, (Madrid: Editorial Universitaria Europea, 1965), 29-59. Adolfo Maíllo, «Concepto y límites de la educación sanitaria escolar», Vida Escolar, n. ${ }^{\circ} 26$ (1961): 2-8. 
autores explica que las propuestas tan solo tuviesen un carácter de declaración de intenciones en las que se mezclaban versiones clásicas de la educación sanitaria con propuestas que la entendían como una herramienta para alcanzar cambios sociales.

La educación sanitaria, por tanto, estuvo planteada desde un cierto "arbitrismo» médico. Jubert, buen conocedor de las ideas sobre educación sanitaria de la OMS y la UNESCO, señaló sobre el desarrollo de actividades al respecto: "cal previament "diagnosticar" l'estat d'“instrucció sanitària" de la població o de grups d'individus, per tal de permetre elaborar el "tractament educatiu" necessari. Però -així mateix- cal saber administrar i dosificar aquest tractament i saber qui l'ha d'administrar». ${ }^{82}$ No cabe una metáfora más apegada a la vertiente asistencial, en un contexto en el que se abjuraba, precisamente, del limitado abordaje de lo curativo.

Desde este punto de vista médico-céntrico, la necesidad de realizar un diagnóstico previo a la acciones educativas, ya propugnado por autores como Mállo y Serigó, desvelaba una valoración ambigua de lo cultural, que muestra la incomodidad en la que también se movía la OMS.83 Aunque se debían conocer y respetar las creencias de la población, habitualmente se valoraban como impedimentos para una educación sanitaria gobernada por profesionales de la salud, en el contexto de un planteamiento clásico basado en la erradicación de determinadas conductas nocivas, o potencialmente dañinas. Hubo, no obstante, propuestas novedosas, como la necesidad de tomar en consideración las «medicinas marginales», que metodológicamente podrían tratarse: «[...] desde una óptica de recuperación y depuración de numerosas costumbres sanitarias que cada niño (y cada enseñante) aporta, en función de su origen familiar y geográfico». ${ }^{84}$ Otras propuestas metodológicas del mismo texto señalaron que la educación sanitaria podría ser abordada desde diversas disciplinas del currículo escolar, evitando una presentación

\footnotetext{
82 Jubert i Gruart, «Educació sanitària: pressupòsits bàsics per una pedagogia de la salut», 347.

83 Organización Mundial de la Salud, Comité de expertos en formación del personal de sanidad para la educación sanitaria popular. Serie Informes Técnicos, 156 (Ginebra: Organización Mundial de la Salud, 1958), 4.

${ }^{84}$ Josep Maria Antó y Josep Martí. «Algunas posibilidades de actuación sanitaria en la escuela»: 68.
} 
dogmática de la «medicina oficial» y subrayando la responsabilidad personal en el cuidado de la salud. ${ }^{85}$

Los educadores y el ámbito escolar como escenario de la educación sanitaria se consideraban imprescindibles. Sin embargo, aparecen llamativas afirmaciones como: «[...] l'organisme directament responsable dels serveis de salut a les escoles ha d'esser 1'hospital [...]»,86 referida a todos los espacios de la sanidad escolar: higiene, prevención, asistencia y curación de las patologías escolares, y educación sanitaria. Hay que contextualizar esta afirmación, y otras similares, en el marco de la deficiente situación de la sanidad escolar, cuya muy insuficiente reforma provisional se había acometido poco antes de la muerte del dictador ${ }^{87}$ En otros casos la preponderancia del escenario sanitario en la sanidad escolar se trasladó a contextos locales, como comarcas, municipios o barrios. ${ }^{88}$

En los escritos específicos sobre educación sanitaria se incidió de nuevo en considerarla como una herramienta para la participación de la población en la gestión de un sistema sanitario democrático, cuyo objetivo sería un nuevo tipo de sociedad con la salud colectiva como aspiración primordial. Sin embargo, algunas de estas ideas no partieron de profesionales de la salud pública, sino, mayoritariamente de clínicos, lo que explica no pocas paradojas de lo propuesto.

La reiterada alusión a la dimensión política de la educación sanitaria, que exigía reivindicaciones populares sobre el sistema sanitario rara vez fue atada a tierra:

En definitiva, pensamos que hoy ya es posible ir construyendo una pedagogía sanitaria en las escuelas, a partir de la comprensión del hombre como una realidad psico-somática-social, cuyas

\footnotetext{
${ }^{85}$ Los únicos textos que se ocuparon con detalle de programas de educación sanitaria, pero todavía fuera del contexto reformador fueron: Yuste Grijalba, La educación sanitaria y Yuste Grijalba, Hacia una sociología de la medicina.

86 Martí i Valls, «La sanitat i l'escola», 362. En el mismo sentido se pronunciaron Casares y Roca, «La medicina i la higiene escolar», 163: «L'organització de la sanitat escolar ha de correspondre, doncs a la Conselleria de Sanitat, o més específicament, al Servei Nacional de Salut, que planificarà la protecció a tots els infants en un sistema únic i gratuït».

${ }^{87}$ Decreto 2892/1975, de 31 de octubre, por el que se aprueba el Reglamento Provisional de Sanidad Escolar. Boletín Oficial del Estado de 13 de noviembre de 1975, n. ${ }^{\circ} 272:$ 23687-23692.

${ }^{88}$ Casares y Roca, «La medicina i la higiene escolar».
} 
enfermedades son inseparables de su personalidad individual y colectiva, y de la formulación de una política de defensa de la salud ligada a las iniciativas de la base social, que considere la globalidad de recursos sanitarios y que señale a cada persona como el portador y gestor de su propia salud. ${ }^{89}$

Jubert, por sus variados intereses en torno al desarrollo del niño, la educación sanitaria, la educación especial y otros campos de saber, como la historia del arte, se separó del camino trillado y fue más allá de las preocupaciones higiénicas clásicas, criticadas, pero omnipresentes..$^{90}$ Señaló que en el contexto de un nuevo sistema sanitario la educación sanitaria "clásica», centrada, básicamente, en la enseñanza de la higiene, pasaba a ser «educación para la salud», a partir de un determinado concepto de salud, que en el contexto catalán era el de Gol. ${ }^{91}$ Indicó que en su órbita debían de estar temas como: el respeto a la vida humana e individual, el rechazo del dualismo mente-cuerpo, conocimientos sobre las conductas, sentido de la vida y de la muerte, asunción de las limitaciones de la medicina, actitud crítica frente al consumismo general y sanitario. La originalidad de las propuestas no se acompañó de indicaciones sobre cómo afrontar temas tan complejos. Parece más bien un desiderátum sin traducción en acciones concretas, como ocurrió en la mayoría de los escritos que venimos comentando.

La importancia de la educación sanitaria comenzó a plasmarse en escenarios sanitarios locales a finales de los años setenta. En este proceso desempeñó un papel relevante una institución de referencia, el Centro Sperimentale per l'Educazione Sanitaria de Perugia, creado a mediados de los años cincuenta. Muchos sanitarios españoles viajaron a la ciudad italiana para formarse en sus cursos de verano y, posteriormente,

\footnotetext{
89 Josep Maria Antó y Josep Martí. «Algunas posibilidades de actuación sanitaria en la escuela»: 69. Años después, uno de los autores de esta afirmación presentó la tesis Josep Martí i Valls. Moviments socials i reivindacions sanitàries a Catalunya (1970-1976). (Tesis Doctoral, Universitat Auntònoma de Barcelona, 1981), en la que analizó las iniciativas de base social a las que se refiere la cita.

90 Sí se incluyó, en la mayoría de las propuestas, la educación sexual, el ámbito en el que se produjeron propuestas pedagógicas más novedosas. Para un análisis detallado del tema véase: Inma Hurtado García y Aida Terrón Banuelos, «La educación sexual durante la Transición: Modelando discursos y modulando voces», en eds. José Martínez-Pérez y Enrique Perdiguero-Gil, Genealogías de la reforma sanitaria, (Madrid: Catarata, 2020), 155-191.

${ }_{91}$ También aparece este tránsito terminológico y conceptual en: "Comissió d'Educació Sanitaria», 86-89.
} 
algunos profesores de este centro se desplazaron a España para dar cursos. ${ }^{92}$ Fue en centros municipales y, sobre todo, en los centros de Atención Primaria donde empezó a tener carta de naturaleza la educación sanitaria (ahora educación para la salud) que se había propugnado a nivel genérico. Los profesionales de Enfermería tuvieron un especial protagonismo en este proceso. ${ }^{93}$

\section{CONCLUSIONES}

La puesta en marcha de la Seguridad Social en España se produjo en 1967, tras la larga gestación de los principios establecidos en la Ley de Bases de la Seguridad Social de 1963. El nuevo esquema no solventó muchos de los problemas que se había propuesto, aunque sí aumentó sensiblemente el porcentaje de población con cobertura sanitaria. Apenas variaron las líneas principales de la asistencia, establecidas desde la instauración del SOE: atención fundamental a lo curativo frente a la prevención, postergación de las dimensiones colectivas de la salud y la enfermedad, medicina general de baja calidad y centralidad de la receta ante la inexistencia de una relación asistencial técnicamente correcta. La hegemonía de la atención hospitalaria se acrecentó con el desarrollo de un determinado modo de conceptuar la medicina, muy biologicista, y la proliferación de nuevas instalaciones nosocomiales. Esta situación supuso que, a mediados de los años setenta, hubiera un consenso general, tanto en el gobierno, como en las clandestinas fuerzas políticas y sindicales, sobre la necesidad de modificar el sector sanitario. Claro está, los parámetros sobre los que debía pivotar tal tarea variaban, algunos hablaban de reformar el sistema sanitario y otros de edificar uno nuevo. Sin embargo, todos los escritos que hemos analizado, surgidos desde diferentes instancias, coincidieron en considerar a la educación sanitaria como un elemento capital. Del mismo modo, coincidieron en el diagnóstico de la situación previa: nada, o casi nada, se había hecho al

\footnotetext{
92 Josep M. Comelles et al., «Health education and medical anthropology in Europe: the cases of Italy and Spain», Salud Colectiva 13, n. 2 (2017): 171-198, https://doi.org/10.18294/sc.2017.1196. Duro Martínez, «Discursos médicos y políticos».

${ }_{93}$ María Jesús Pérez Mora, «La reforma de la Atención Primaria de Salud: sus inicios en la Comunidad Valenciana», en Política, salud y enfermedad en España: entre el desarrollismo y la transición democrática, ed. Enrique Perdiguero Gil (Elche: Universidad Miguel Hernández, 2015).
} 
respecto durante el franquismo. Las fragmentarias iniciativas desarrolladas no merecieron, ni siquiera, el papel de «antecedentes».

Las propuestas que pretendieron poner en marcha un servicio nacional de salud situaron a la educación sanitaria de la población en la base de un nuevo sistema que primase la defensa de la salud frente al tratamiento de la enfermedad, ofreciendo atención integral, con cobertura universal, financiado a cargo de los presupuestos generales del estado, descentralizado y con participación de la población en la gestión y planificación. Los proyectos reformadores gubernamentales, reticentes a abandonar el sistema de aseguramiento y con menos énfasis en aspectos como la descentralización y la cogestión, compartían, sin embargo, otros muchos rasgos con los rupturistas.

Una de esas coincidencias, aun con notables variaciones de matiz, fue la consideración de la educación sanitaria de la población desde una perspectiva ingenua - una especie de panacea sin mayor concreción-, clásica — consejos a la población en torno a conductas no saludables, en especial a grupos vulnerables específicos-, y con mucho énfasis en su papel de guía para un uso "correcto» de los servicios sanitarios dentro de los parámetros establecidos por los expertos. Este planteamiento predominante de la educación utilizó una cierta retórica de relación con el sistema educativo, pero, en la mayoría de los casos, sin mayor pretensión de colaboración.

En conjunto, puede afirmarse que la importancia dada a la educación sanitaria de la población fue tan grande, como ingenuo, clásico e inconcreto su planteamiento, y con una mayoritaria consideración del papel hegemónico de la medicina a la hora de guiar los derroteros de este tipo de actividades. Solo de manera incipiente, a final de la década de los setenta, algunos profesionales, a través de iniciativas creativas - muchas veces de cariz municipal_-, y/o debido a la influencia de corrientes internacionales, fueron poniendo las bases de lo que sería una «educación para la salud» desarrollada de manera más concreta, sistemática y consciente durante las dos décadas siguientes.

Cabría plantear una última pregunta: ¿Persiste en la actualidad este enfoque heredero de los anhelos de un nuevo modo de afrontar la salud y la enfermedad que se expresaron durante la Transición democrática? 
Si cualquier ciudadano visitase hoy un centro sanitario, sea del nivel que sea, ¿identificaría fácilmente actividades que sustentasen ese papel clave que se quiso otorgar a la educación sanitaria de la población? La respuesta requeriría el desarrollo de todo un programa de investigación que todavía no se ha afrontado globalmente.

\section{Nota sobre los autores}

Enrique Perdiguero Gil es doctor en Medicina por la Universidad de Alicante. Catedrático de Historia de la Ciencia en la Universidad Miguel Hernández de Elche. Ha realizado varias estancias de investigación en el Wellcome Institute for the History of Medicine (Londres), del que fue Research Fellow. Sus líneas de investigación se han centrado las relaciones entre conocimiento científico y conocimiento popular, prestando especial atención a la popularización de la medicina y la educación sanitaria, desde una perspectiva que aúna la antropología social y la historia de la medicina. También se ha ocupado de la historia de la Salud Pública en España, de la historia de la Antropología Médica en España y de la historia del pluralismo asistencial. El resultado de estas investigaciones lo ha dado a conocer en libros, capítulos de libros y artículos publicados en revistas especializadas. Ha participado en numerosos congresos nacionales en internacionales. En los últimos años ha dirigido proyectos de investigación relacionados con la educación sanitaria en el Franquismo, el desarrollo del Seguro Obligatorio de Enfermedad, la medicina rural durante el Franquismo y la reforma sanitaria en la Transición Democrática. Ha sido miembro de la junta directiva y vicepresidente de la Sociedad Española de Historia de la Medicina y miembro de la junta directiva de la Societat Catalana d'Història de la Ciència i de la Tècnica. Actualmente dirige la sede del Instituto Interuniversitario López Piñero de Estudios Históricos y Sociales, sobre ciencia, tecnología, medicina y medio ambiente en la Universidad Miguel Hernández de Elche.

Eduardo Bueno Vergara es doctor en Historia por la Universidad de Alicante y Máster en Historia de la Ciencia y Comunicación Científica (Universidad de Alicante, Universidad Miguel Hernández de Elche y Universidad de Valencia). Es profesor ayudante doctor en el Área de 
Historia de la Ciencia de la Universidad Miguel Hernández de Elche e investigador del Instituto Interuniversitario López Piñero de Estudios Históricos y Sociales, sobre ciencia, tecnología, medicina y medio ambiente. Sus principales líneas de investigación son la historia del clima, la historia de la salud y la enfermedad durante la dictadura franquista y la historia del cáncer durante el mismo periodo. Sobre estos temas ha publicado diversos capítulos de libros y artículos en revistas especializadas.

\section{REFERENCIAS}

Acarín, Nolasc. «Bases para la regionalización sanitara». En La sanidad hoy. Apuntes críticos y una alternativa, editado por Nolasc Acarín, Ramón Espasa, Helios Pardell, et al., 111-133. Barcelona: Avance, 1975.

Acarín, Nolasc, Manuel Campo, Ramón Espasa, y Joaquim Vergés. La salud, exigencia popular. Barcelona: Laia, 1976.

Acarín, Nolasc, Ramón Espasa, Carme Sans y Joaquim Vergés. Servicio Nacional de Salud. Una alternativa democrática a la sanidad. Barcelona: Laia, 1977.

Bernabeu-Mestre, Josep y Encarna Gascón Pérez. Historia de la Enfermería de Salud Pública en España (1860-1977). Alicante: Publicaciones de la Universidad de Alicante, 1999.

Bernabeu-Mestre, Josep y María Eugenia Galiana Sánchez. «Enfermería y exilio. El caso de las visitadoras sanitarias». Mètode, n. 61 (2009): 72-77.

Bonal, Joaquim. «Farmàcia e industria farmacèutica». En Xè Congrés de Metges i Biòlegs de Llengua Catalana. II Ponència. Funció social de la medicina, 384398. Barcelona: Acadèmia de Ciències Mèdiques de Catalunya i de Balears. Societat Catalana de Biologia, 1976.

Borasteros, Carlos. Salud, enfermedad y sociedad. Madrid: Forma, 1978.

Bueno Vergara, Eduardo y Enrique Perdiguero-Gil. «Mejor curar que prevenir: dispositivos asistenciales y actividades preventivas en el primer franquismo». En Del siglo XIX al XXI. Tendencias y debates, editado por Mónica Moreno Seco, Rafael Fernández Sirvent y Rosa Ana Gutiérrez Lloret, 19721983. Alicante: Biblioteca Virtual Miguel de Cervantes, 2019.

Campos i Avillar, Joan. «Hacia un modelo de ruptura educativa para la reforma sanitaria». En Planificación y reforma sanitaria, editado por Jesús M. de Miguel Rodríguez, 121-151. Madrid: Centro de Investigaciones Sociológicas, 1978.

Casares, Ramon y Francina Roca. "La medicina i la higiene escolar». En Salut, sanitat $i$ societat. Per una resposta socialista a l'actual situació sanitària, 
editado por Jordi Gol i Gurina, Josep Artigas Candela, Joaquim Jubert i Grurart, et al., 155-175. Barcelona: 7 x 7 edicions, 1977.

Castejón Bolea, Ramón, Enrique Perdiguero Gil y José Luis Piqueras Fernandez, eds. Las imágenes de la salud: cartelismo sanitario en España (19101950). Alicante: Instituto Alicantino de Cultura «Juan Gil-Albert». Consejo Superior de Investigaciones Científicas, 2012.

Castejón, Ramón, Enrique Perdiguero y Rosa Ballester. «Los medios de comunicación al servicio de la lucha antivenérea y la protección de la salud maternoinfantil (1900-50)». História, Ciências, Saúde-Manguinhos 13, n. 2 (2006): 411-437.

Comelles, Josep M., Isabella Ricco, Aida Terrón Bañuelos y Enrique Perdiguero-Gil. "Health education and medical anthropology in Europe: the cases of Italy and Spain». Salud Colectiva 13, n. 2 (2017): 171-198. https://doi. org/10.18294/sc.2017.1196.

De Miguel Rodríguez, Jesús M. «Policies and politics on the health reforms in Southern European Countries: a sociological critique». Social Science and Medicine 11 (1977): 379-393.

De Miguel Rodríguez, Jesús M. La sociedad enferma: las bases sociales de la política sanitaria española. Madrid: Akal, 1979.

Duro Martínez, Juan Carlos. «Discursos médicos y políticos sobre la salud comunitaria durante la transición democrática española». Praxis Sociológica, n. ${ }^{\circ} 18$ (2014): 35-79.

Echeverri Dávila, Beatriz. «La protección de la infancia: la educación sanitaria de las madres en la posguerra española». Historia y Política, n. 9 (2003): 279-308.

Evangelista Benítez, Manuel. Medicina y sociedad: la reforma sanitaria. Madrid: Ministerio de Trabajo y Seguridad Social, 1981.

Galiana Sánchez, María Eugenia, María Pilar García Paramio y Josep Bernabeu Mestre. «La frustración de una profesión: la enfermería de salud pública en la V Reunión de Sanitarios, 1959». Temperamentvm 5, n. 9 (2009).

García i Balda, Josep María. «Aproximació històrica a l’educació especial a Catalunya: l'educació institucionalitzada en centres d'educació especial a les comarques de Girona (1873-1997)». Tesis Doctoral, Universitat de Girona, 2007.

García Jiménez, María Teresa. «Introducción. Breve historia de la alimentación y la nutrición en los colegios españoles. Recursos y currículo». En Nutrición y Alimentaciónn en el ámbito escolar, editado por Jesús Román Martínez Álvarez, 1-21. Majadahonda, Madrid: Ergon, 2012.

Gol i Gurina, Jordi, Jesús M. de Miguel, Joan Reventós, Andreu Segura y Félip Solé Sabarís. La sanitat als Països Catalans. Crítica i Documentació. Barcelona: Edicions 62, 1978. 
González García, Alberto. «La Sección Femenina y las campañas de vacunación obligatoria contra la difteria en España en las páginas de La Vanguardia (1940-1955)». Vínculos de Historia. Revista del Departamento de Historia de la Universidad de Castilla-La Mancha 5 (2016): 309-329. https://doi.org/10.18 239/vdh.v0i5.018.

Hurtado García, Inma y Aida Terrón Banuelos. «La educación sexual durante la Transición: Modelando discursos y modulando voces». En Genealogías de la reforma sanitaria, editado por José Martínez-Pérez y Enrique Perdiguero-Gil, 155-191. Madrid: Catarata, 2020.

Infante, Alberto, ed. Cambio social y crisis sanitaria. (Bases para una alternativa). Madrid: Ayuso, 1975.

Lamarre, Marie-Claude. «The International Union for Health Promotion and Education». Health Education Research 15, n. 3 (2000): 243-248. https://doi. org/10.1093/her/15.3.243.

Maceiras-Chans, José Manuel, María Eugenia Galiana-Sánchez y Josep Bernabeu-Mestre. «La Sección Femenina en las campañas de vacunación del primer franquismo: el caso de la provincia de Valencia (1941-1958)». RECIEN 13 (2017): 5-23. https://doi.org/10.14198/recien.2017.13.02.

Maceiras-Chans, José Manuel, María Eugenia Galiana-Sánchez y Josep Bernabeu-Mestre. «Enfermería y control social: las actividades socio-sanitarias de la Sección Femenina de Falange en la ciudad de Valencia (1940-1977)». Enfermería Global 17, n. 1 (2018): 270-282. https://doi.org/10.6018/eglobal.17.1.263381.

Marset Campos, Pedro, José Miguel Sáez Gómez y Fernando Martínez Navarro. «La Salud Pública durante el franquismo». Dynamis 15 (1995): 211-250.

Martí i Valls, Josep. "La sanitat i l'escola». En Xé Congrés de Metges i Biollegs de Llengua Catalana. II Ponència. Funció social de la medicina, 361-364. Barcelona: Acadèmia de Ciències Mèdiques de Catalunya i de Balears. Societat Catalana de Biologia, 1976.

Martínez Navarro, Ferran. Estructura i malatia. Una alternativas sanitària per al País Valencià. València: Tres i Quatre, 1978.

Martínez-Pérez, José y Enrique Perdiguero-Gil, eds. Genealogías de la reforma sanitaria. Madrid: Catarata, 2020.

Mayor Domingo, Federico, ed. Investigación sobre la asistencia farmacéutica en España: estudio socioeconómico sobre el conjunto de la asistencia sanitaria española. Madrid: Ministerio de Trabajo. Instituto Nacional de Previsión, 1977.

Nadal, Juli. La construcción de un éxito. Así se hizo nuestra sanidad pública. Barcelona: Ediciones La Lluvia, 2016.

Nájera, Pilar. "Health Education in Spain - AN OVERVIEW». En World Yearbook of Educacion. Health Education, editado por Chris James, John Balding y Duncan Harris, 64-72. London: Kogan Page, 1989. 
Novella, Enric J. «Los límites de la tecnocracia: el Patronato Nacional de Asistencia Psiquiátrica y la modernización autoritaria de la asistencia psiquiátrica en la España del segundo franquismo». Dynamis 39, n. 1 (2019): 73 97. https://doi.org/10.30827/dynamis.v39i1.8667.

Palacio Lis, Irene. Mujeres ignorantes: madres culpables adoctrinamiento y divulgación maternoinfantil en la primera mitad del siglo XX. València: Universitat de València, 2003.

Pan-Montojo, Juan. «Entre la reforma y las contrarreformas: una historia política de la fiscalidad española en la democracia». En La evolución de la Hacienda Pública en Italia y España (siglos XVIII-XXI), editado por Carlos Barciela López, Antonio Di Vittorio y Joaquín Melgarejo Moreno, 557-590. Alicante: Publicacions de la Universitat d'Alacant, 2015.

Perdiguero-Gil, Enrique. «Propaganda y “educación sanitaria” en el ideario médico-social del Franquismo a través de la revista SER». En Politica, salud y enfermedad en España: entre el desarrollismo y la transición democrática, editado por Enrique Perdiguero-Gil. Elche: Universidad Miguel Hernández, 2015.

Perdiguero-Gil, Enrique. «La popularización médica durante el franquismo: un primer acercamiento». En Educación, comunicación salud. Perspectivas desde las ciencias humanas y sociales, coordinado por Josep M. Comelles y Enrique Perdiguero Gil, 105-138. Tarragona: Publicacions Univeristat Rovira i Virgili, 2017.

Perdiguero-Gil, Enrique y Josep M. Comelles. «The Roots of the health Reform in Spain». En Health Care and Government Policy, editado por Laurinda Abreu. Évora: Publicações do Cidehus, 2019.

Perdiguero-Gil, Enrique y Josep M. Comelles. «The defence of health. The debates on health reform in 1970s Spain». Dynamis 39, n. 1 (2019): 45-72. https://doi.org/10.30827/dynamis.v39i1.8666.

Perdiguero, Enrique, Rosa Ballester y Ramón Castejón. «Films in Spanish Health Education: The Case of Child Health (1928-1936)». Higyea Internationalis 6, n. ${ }^{\circ} 2$ (2007): 69-97.

Pérez Mora, María Jesús. «La reforma de la Atención Primaria de Salud: sus inicios en la Comunidad Valenciana». En Política, salud y enfermedad en España: entre el desarrollismo y la transición democrática, editado por Enrique Perdiguero Gil. Elche: Universidad Miguel Hernández, 2015.

Pérez Moreno, Heliodoro Manuel. «La asistencia sanitaria cono función circumformativa de la Cátedra Ambulante de Huelva (1956-1977)». Cuestiones Pedagógicas, n. ${ }^{\circ} 21$ (2011/2012): 197-228.

Pérez Moreno, Heliodoro Manuel. «Educación y asistencia social de una escuela errante durante el Franquismo en España». Revista de Educación Social, n. ${ }^{\circ} 17$ (2013): 1-16. 
Rodriguez-Ocaña, Esteban. «La sanidad franquista vista desde la Organizacion Mundial de la Salud: el Informe Brockington (1967)». Gaceta Sanitaria 32, n. 6 (2018): 582-583. https://doi.org/10.1016/j.gaceta.2018.03.004.

Rodríguez Ocaña, Esteban y Juan Atenza Fernández. «El proyecto E30 OMS-España para el establecimiento de una zona de demostraciones sanitarias en Talavera de la Reina, 1965-1976». En Salud, enfermedad y medicina en el franquismo, editado por $\mathrm{M}^{\mathrm{a}}$ Isabel Porras Gallo, Lourdes Mariño Gutiérrez y María Victoria Caballero Martínez, 124-145. Madrid: Catarata, 2019.

Rodríguez Ocaña, Esteban y Rosa Ballester Añón. «El Informe del consultor de la OMS Fraser Brockington de 1967 en el contexto del reformismo sanitario franquista». Dynamis 39, n. 2 (2019): 477-496. https://doi.org/10.30827/dynamis.v39i2.9845.

Salazar Agulló, Modesta, Emilio Martínez Marco y Josep Bernabeu-Mestre. «La salud maternoinfantil durante el franquismo: notas bibliométricas sobre el programa «Al servicio de España y del niño español»». Asclepio 59, n. ${ }^{\circ} 1$ (2007): 285-314.

Sánchez Harguindey, Luis. Reforma sanitaria española. Madrid: Acción Social Empresarial, 1981.

Serigó Segarra, Adolfo. La crisis sanitaria en España (Nuevas ideas en salud pública). Madrid: El autor, 1979.

Trescastro-López, Eva María y Silvia Trescastro-López. «La educación en alimentación y nutrición en el medio escolar: el ejemplo del Programa EDALNU». Revista Española de Nutrición Humana y Dietética 17, n. ${ }^{2}$ (2013): e84-e90. https://doi.org/10.14306/renhyd.17.2.11.

Vanel, Julia «De l'éducation sanitaire à la promotion de la santé: Enjeux et organisation des savoirs au coeur de l'action publique sanitaire (internationale)». PhD. diss., Université Paris Saclay (COmUE), 2006.

Vicarelli, Giovanna. «The creation of the National Health System in Italy (19611978)». Dynamis 39, n. ${ }^{1}$ (2019): 21-43. https://doi.org/10.30827/dynamis. v39i1.8665.

Vilar-Rodríguez, Margarita y Jerònia Pons-Pons. «The Introduction of Sickness Insurance in Spain in the First Decades of the Franco Dictatorship (19391962)». Social History of Medicine 26, n. 2 (2012): 267-287. https://doi.org/ 10.1093/shm/hks082.

Yuste Grijalba, Francisco Javier. Hacia una sociología de la medicina. Barcelona: Aula de Ediciones, 1971.

Yuste Grijalba, Francisco Javier. La educación sanitaria. Madrid: Marsiega, 1971. 